ISSN 1112-9867

http://www.jfas.info

\title{
A STUDY ON THE PERSONALIZATION METHODS OF THE WEB
}

\author{
M. Hajighorbani*, S. M. Reza Hashemi, M. M. Deramgozin and M. Faridpour
}

Young Researcher and Elite Club, Qazvin Branch, Islamic Azad University, Qazvin, Iran

Published online: 19 June 2016

\begin{abstract}
Search engine personalization is one of the various deep personalization methods. It can be said that personalization systems that help users find the information they need requires the use of contextual and semantic information analysis techniques that exist in the field of data recovery such as web personalization and the process of optimizing the methods to get to web pages in a way that are consistent with the needs of each user. What helps the current problem of search engines and accelerate their performance, is providing a proper framework for finding the correct pattern considering great items in history of users. This approach improves the advising process of the search engines as well. The aim of this paper is to introduce some process improvement methods of correct patterns and analyze them. Here we will discuss the basic concepts of web personalization and consider the three approaches of web personalization and we evaluated the methods belonging to each of them.
\end{abstract}

Key words: personalization, search engine, user preferences, data mining methods.

Author Correspondence, e-mail: mhajighorbani@comp.iust.ac.ir

doi: http://dx.doi.org/10.4314/jfas.8vi2s.49

\section{1- INTRODUCTION}

Nowadays the exponential growth and change in the volume of web information causes users not to have quick and easy access to the information they need. Researcher's problem today is not 
lack of resources but rather expanding volume of information has been a controversial challenge. Finding relevant information and quality among the mass of the topics released in various formats as well, is another challenge. Although the use of tools such as search engines can help users find the information they need, enabling and facilitating access to web information, the amount of information that search engines provide for the users is usually much more than the amount to be quickly processed by the user. In addition, most search engines use the content of the pages and the links between them to determine the relevance of pages with user's query. In fact, search engines do not consider user's interests and background knowledge in searches, but recover the same results for users with different interests and same queries. Therefore considering the importance of rapid access to relevant information for users interacting with the web, web personalization has become a popular phenomenon in order to customize the web environment and plays an important role in providing quickly access and needed information by users according to their interests. Personalization the results of web search engines are of particular importance. Search engines such as yahoo and Google-based keywords are the main tools to use web today. It is clear that web search engines could not achieve great success without considering search engines. However there are serious problems in relation to their use as following:

1. High recovery, less accuracy; even if the main pages, retrieved; it is not possible to use them well because the great number of pages of information retrieval, can be as bad as too few of them.

2. Low or zero recovering; it often happens that search engine does not provide relevant answers for the query terms or important pages cannot be retrieved.

3. The results are very sensitive to keywords; initial key words often do not lead to desired outcome. In this case related documents include words different from the query. This causes discontent because queries with the same meaning have to restore similar answers. It is interesting that despite the progress that has been made in the search engine technology, the problem still remains. Providing a logical trend, in this article we're going to classify user behavior and label them in a proper manner. 


\section{1- The methods of classification}

\section{1-1- Web personalization}

Measures that make the provided information or services by a web server compatible with user needs considering the history of users' workflow and personal interests are called web personalization [5]. The goal is to provide the desired and required information without users' explicit request.

\section{1-1-1 Advantages}

1. Personalization of services plays an important role in reducing traffic and makes the internet a user-friendly environment for people.

2. By providing user-desired information in a reasonable manner and at the right time improves the circulation of the user on the website.

3. In e-commerce provides a mechanism to better understand customer needs, identifying future trends and ultimately increases the loyalty of customers to offered service.

\section{1-1-2- different web personalization operations}

The web personalization operations are divided into several categories [6]:

\section{- Notation}

Information about the user, such as name and his work history is stored in the system. When the user logs in again, this information can be used without any processing. Among these operations are:

1. Welcome the user

2. Mark the pages for the user

3. Personalized rights of access

\section{- Help}

Operations with the aim of quick recovering of information needed by user in the web and also providing alternative suggestions are in this category. As some instances:

1. Recommended link

2. User training 


\section{- Adaptation and adjustment}

Content, structure and layout of the user are replaced with the knowledge, preferences and interests of the user. As some instances:

1. Adjusting the content

2. Adjusting the links

3. Personalized pricing

4. Personalized distinction among the products.

\section{- Support for task execution}

Implementing a particular operation by the user without his knowledge is called supporting task execution. As some instances:

1. Personalized messaging

2. Personalized question completion

3. Personalized discussion

\section{1-1-3- Operational system requirements}

Operational requirements are considered in system design personalization. These requirements include:

The domain specifications: Functions provided by the system, is sensitive to the domain.

Therefore the domain specifications must accurately be described.

User identification: The system is in direct interaction with the user and is aware of his behavior. An appropriate mechanism should exist to identify and differentiate users.

Monitor the users' data: The system must be able to collect all data related to users.

Data preparation: data collected should be pre-processed in order to eliminate noises and be converted into a suitable format.

Build the user's model: the main component of the system, is the user that includes information about the interests, knowledge, goals and preferences of the user. Modeling can be done manually or automatically. 
Privacy issues: the user privacy must be protected at all times and the user should be aware of how to collect and use it.

\section{1-1-4 Personalization approaches}

Existing systems can be categorized in three approaches [7]:

\section{- Decision based systems}

According to this method, a web service is personalized through direct involvement of the designer and usually with the assistance of the user. These models are usually static and a number of rules are determined directly about the way of delivering web content to users with different models. These systems require the great volume of activities directly for the construction and maintenance. In addition they usually require user interaction, which is not desirable in personalization.

These systems use user profiles and recommend pages with new items based on the similarity of their content with pages and items that are available in user profile. Common mechanism in these systems are usually comparing the key words representing pages or describing items and are not useful in e-commerce applications because on one hand website owners have less control on users and on the other hand techniques based on content similarity alone usually does not consider other semantic relationships between objects.

\section{- Limiting social or collective systems}

The main focus of these systems is based on similarity between users rather than similarity of more items. These systems compare all user recorded preferences to the history of other users to find users with similar interests. This set of users is called users with similar interests with the neighborhood user. Mapping the history of a user to its neighbors can be on the basis of ranking similarity of items, access to pages with similar content or purchase similar items.

\section{1-2 Web mining process steps}

The general web personalization process based on web mining consists of three steps:

1. Data preparation (data preprocessing) 
2. Discovering a pattern in the data prepared by the previous stage (modeling)

3. Analysis of obtained patterns and use the discovered patterns to customize the web takes place in the first phase of initial processing of the data. In the second phase, statistical methods including methods for data mining is used to detect the desired pattern.

\section{1-2-1- Information gathering}

As mentioned, information about the behavior of user is gathered through different sources such as reporting on a server, client reports and personal information of organization or the combination of above mentioned resources.

\section{1-2-2- Data preparation}

An important step in any data-mining activities is to create a good data set to apply data mining algorithms on it. This process can include pre-processing raw data, integrate data from various sources and convert integrated data into the right format as the input for data mining operations. This operation is called data preparation. Web usage data specifies user profile and their behavior in the web survey. These data are usually collected in three ways:

- Web servers

- Proxy servers

- Web clients

\section{1-2-3- Data preprocessing}

At this point usage, content and structure data derived from a variety of data sources include various and useless data. This information should be classified and in order to be used in pattern recognition stage.

Data preprocessing include information deleting stages and adding additional irrelevant information. User recognition and session are the main activities done in the pre-processing of information and this is one of the important activities before pattern recognition stage. Accuracy is essential at this stage because if this stage is incomplete the pattern recognition stage will be faced with difficulties. The result is influenced by this stage.

Preprocessing is done on the content, profile and structural data. 
Preprocessing log files is complex and time-consuming. For this purpose, usually four stages are taken:

\section{Erasing data}

For data cleaning, all data stored in log files that are not used in the process of exploring will be deleted.

\section{Detect and reconstructing user sessions}

Detection and session reconstruction is made up of two steps. Recognizing the different user sessions based on weak data existing in log files, and restore the user's path was diagnosed in recognized sessions.

\section{Recover information about the content and structure of pages}

It has always been tried to use the information contained in web pages in explorations. In some of methods related to web pages searches, web pages are classified based on their content and this information is utilized to strengthen the information contained in log files.

\section{Data formatting}

For this purpose, extracted data from the log files can be added to relational databases so different queries can be easily implemented on them. Additional and useless information is separated from the rest of the information at this stage and gathered information change to integrated information that can be used as input for the next step to pattern discovery. One of the important tasks done in this step is eliminating additional information and detecting the duration of user's profile.

\section{4-2-1- Transaction detection}

In some applications, user sessions can be processed to produce suitable elements. One of these elements is the concept of transaction or course. A transaction is a subset of the linked pages that have occurred in a user session. Identify transactions is strongly dependent on search behavior of a particular user on the website and can be identified by the use of conceptual information. Transactions are divided into two categories:

1. Content only transactions correspond to pages visited by the user.

2. Content-circulation transactions corresponding to routes leading to content pages. 
Conceptual information is usually used in order to identify a sequence of actions that are related to a specific font and are utilized on the trail of the user's browsing domain. Actions performed without changing, form the local history and are used to analyze the behavior of the user.

At the end of this stage we have reached a transaction-type dataset that each row will display the history of a particular user and each column is related to features of the examining page.

This approach has a fundamental flaw and that is the recommendation to the user is only on the basis of his existing transactional data and hence most recently added items or issues cannot be recommended to him. This problem is commonly called the new pen problem. On the other hand, although the previously discovered patterns on the web are useful to find the relation of items with each other or with the user, provide little understanding about the reasons why items or users are placed together in groups. A common approach to solve this problem is mass filtering that users with the same interests are combined with rankings and judgments of users. In general, keywords in this approach are extracted from the content of the website and used for indexing pages based on the content and categorizing them to different classes. In the field of web personalization this approach allows the system to recommend pages to the user not only based on similar people, but also based on their content similarity to recent pages.

\section{1-2-5- Pattern recognition}

Nowadays, due to the spread of information it is necessary to use techniques such as data mining in order to extract hidden knowledge in the data. Data-mining means finding hidden patterns in available data sets. One of the most important and widely used data-mining techniques to discover hidden knowledge is exploration of association rules. Discovering useful rules is a source of useful information for people working in various fields including trade helping them to make decisions.

At this point an appropriate model is presented for the users' behavior to make a better understanding of the users' behavior. At this point our knowledge about users' behavior patterns generally is obtained by machine learning algorithms or statistical algorithms. For example, the 
clustering, classification, dependence association and sequential patterns discoverer van be mentioned.

\section{1-2-6 presenting the results to the user}

Finally, the knowledge gained from previous stages will be useful and understandable to us that can be in the form of reports and charts or be utilized in other programs to provide services to another.

\section{1-3 pattern discovery techniques}

At this stage machine learning algorithms and statistical methods are used to obtain user behavior patterns that can be divided into four categories:

1. Clustering

2. Classification

3. Association discovery

4. Sequential pattern discoverer

\section{1-3-1- Clustering}

Clustering means putting information sets in different clusters so that they are similar and the information of different clusters are different from each other. Clustering algorithms are categorized in the following groups:

1. Hierarchical methods

2. Partition methods

3. Model-based methods

\section{1-3-1-1- Hierarchical methods}

The input of the algorithm is only the set of samples and similarity criterion. The output is a hierarchical structure of categories. Hierarchical classification algorithms are divided into two categories. The main point in hierarchical clustering is continuous development of clusters. In this type of clustering, data is displayed as a graph that there are two ways to build these graphs: 
- Bottom-up approach: In this method that is also called cumulative, each data is considered as an only element cluster and then in succession the closest cluster or in other words most similar clusters are merged together. This process will continue until we achieve a single cluster or a predefined threshold is met.

- Top-down approach: In this method that is also known as the divisor method, first the entire data set is considered as a single cluster and then successively is divided into smaller clusters.

\section{1-3-1-2 Partition method}

This type of clustering is also called the objective function-based clustering. The foundation of clustering based on segmentation is a certain objective function that its minimization leads to the discovery of the structure of clusters in the data set. The number of clusters is pre-determined or dynamically determined. This way, data are divided into $\mathbf{c}$ clusters so that each cluster contains the following features:

1. All clusters are non-empty and also do not contain all the data.

2. Each data belong to only one cluster.

In this way of clustering, the clusters will appear in two forms:

1- A set of initial points selected as the center of the cluster displayed by $C_{1}, C_{2}, \ldots, C_{k}$.

2- Partition matrix of $U=\left[u_{i j}\right]$ composed of $\mathbf{C}$ rows and $\mathbf{N}$ columns. $\mathbf{C}$ equals with the number of clusters and $\mathrm{N}$ equals with the number of data. Matrix elements show the allocation of corresponding data to clusters.

There are several methods for formulating the objective functions. Criterion of minimizing the variance is one of the most frequently used methods for data clustering. If $\mathbf{N}$ is the number of data and $\mathbf{c}$ is the number of clusters, the total dispersion between data and $\mathrm{C}_{1}, \mathrm{C}_{2}, \ldots, \mathrm{C}_{\mathrm{k}}$ is calculated as follows: 


$$
Q=\sum_{i=1}^{c} \sum_{j=1}^{N} u_{i j}\left\|x_{j}-c_{i}\right\|^{2}
$$

where, $\left\|x_{j}-c_{i}\right\|$ is the Euclidean distance between $\mathbf{x}_{\mathbf{j}}$ and $\mathbf{c}_{\mathbf{i}} \cdot \mathrm{U}=\left[\mathrm{u}_{\mathrm{ij}}\right]$ is the segmentation matrix. If the $\mathrm{j}$ th data belongs to the $\mathrm{i}$ th cluster, $\mathrm{u}_{\mathrm{ij}}=1$ and if notu $\mathrm{u}_{\mathrm{ij}}=0$.

$\mathrm{Q}$, is the objective function and minimizing it makes a segmentation matrix and a set of clusters. To optimize Q, there are many ways that the most important ones include K-Means algorithm and Fuzzy C-Means algorithm.

\section{1-3-1-3- model based approaches}

This category of approaches considers a model for each class and based on the model assumed samples that best match the models are ascribed to that cluster. For example an algorithm based on the model may show the classes through creating a density function that show the distribution of samples in space.

\subsubsection{Classification}

Unlike categories, here we have predefined classes. Putting the components that we want to classify in these classes we obtain a better understanding of these classes. In exploring the web using classification for modeling the users' behavior when visiting web pages is used. We can divide users into different categories. For example, there are users who are interested in training pages or users who read more news. Neural networks, and methods based on Bayes' rule, are methods that are often used for classification.

Other different methods are used considering rough set theory. This method tries to discover the same classes in a data set. A Rough set includes a series of objects that are members of a class and a series of objects that cannot be a member of that class.

\section{1-3-3 Discovering association rules}

One of the important methods of data mining is discovery of association rules. This method can be useful to discover suitable relationships and dependencies that are available in large 
collections of data. One obvious application of association rules is a shopping basket analysis trying to find dependencies and relationships between the goods purchased by the customers and analyze them. For example, customers purchase milk and bread together $[3,4]$.

Suppose that $I=\left\{i_{1}, i_{2}, \ldots ., i_{m}\right\}$ is the set of data items and $m$ is the number of data items. $D$ is the set of transaction data where for each $\mathrm{T}$ transaction there is a unique TID identifier andT $\subseteq \mathrm{I}$. $\mathrm{T}$ transaction includes set item of $\mathrm{A}$ if and only if $\mathrm{T} \subseteq \mathrm{A}$. In other words, every transaction is a subset of items including a unique identifier.

Support: A $\rightarrow$ B law is the proportion of transactions involving A and B to the total number of transactions. In other words, it is the probability that A and B occur together in the whole transactions. Support states the total frequency of law with respect to the total transactions:

$$
\operatorname{Support}(A \rightarrow B)=P(A \cup B)=\frac{A \cup B}{D}
$$

Reliability: A $\rightarrow$ B law is the ratio of the number of transactions including A and B to the transactions including A. Reliability implies the power of law:

$$
\text { Conficence }(\mathrm{A} \rightarrow \mathrm{B})=\mathrm{P}(\mathrm{A} \mid \mathrm{B})=\frac{\mathrm{A} \cup \mathrm{B}}{\mathrm{A}}
$$

The K-item set: The item set that includes k items.

The item set support: is the number of transactions that include the considered item set in transaction data set.

The item set with several repeats: an item set that supporting it makes the minimum support possible.

The input data for an association rules discoverer algorithm are a set of transactions that each transaction is a subset of items including a unique identifier. Transactions can be produced with the help of an external process, or extract data from databases or data storages.

Suppose $\mathbf{I}$ is the data items series and $\mathbf{A}$ and $\mathbf{B}$ are two subsets of data items. The general associative law is $\mathrm{A} \rightarrow \mathrm{B}$ where $\mathrm{A} \subset \mathrm{I}, \mathrm{B} \subset \mathrm{I}$ and $\mathrm{A} \cap \mathrm{B}=\varnothing$. Associative law $\mathrm{A} \rightarrow \mathrm{B}$, represents some events of A and B that has happened simultaneously. There are different criteria to evaluate 
the quality of a rule that based on these strong rules they might be selected among a wide range of possible ones. In other words, by these criteria, useless laws are pruned. One of the most famous and widely used criteria is support and confidence that can be expressed as a percentage. Typically, the minimum amount for the support and confidence is determined by someone who is an expert in the field. An association rule is strong if the minimum values related to support and confidence is satisfied. With a series of transactions, the problem of discovering association rules includes production of association rules that have higher support and confidence than the minimum amount of support and confidence [8].

A discoverer association rules algorithm should be able to produce rules at different levels. Multi-level association rules are generated with the help of top-down exploration. First, strong laws are found in the upper levels of hierarchy and then weaker laws are searched and in lower levels. The methods of multi-level association rules discovery are divided into two main categories:

1- The method based on uniform support that uses the same support minimum threshold in order to produce rules in different levels of the hierarchy.

2- The method based on uniform support that each level of the hierarchy has the specified support minimum threshold and lower levels have lower thresholds [9].

The issue that has been less considered in associative rules is extracting the scarce item set or discovering the rare associative rules. Rare association rules are items that rarely occur in a small number of transactions and are usually pruned in the process of discovering association rules. In other words they are rules with low support and high reliability. Algorithms of discovering associative rules based on the type of values of items, can be divided into three categories:

\section{1-3-3-1-Categorical association rules}

In most algorithms of discovering associative rules transactions are in binary form. This means that for every item in the database tables there are corresponding fields and each record represents a corresponding transaction. If any item exist in a transaction, the value of the corresponding field in the corresponding record will equal with one and otherwise is will be zero. 
However in some cases data items may have several discrete values such as level of education that can be bachelors, masters and doctoral degree. Usually this type of data changes into binary data sets. So that, each pair of item-value is considered as a separate item. The discovery of associative rules discovered in this type of transaction is called binary association rules.

In this case, when the data contains a large number of items, and each item has different values, the total number of fields in the database that should be considered, would be great. So the binary database storage of this data is one of the main problems of the algorithm.

\section{1-3-3-2- Discovery of numeric/quantitative association rules}

It is possible that data items adopt continuous values such as grading that can adopt values from 0 to 20. In this case, methods are provided to detect discrete or binary association rules cannot be used for this type of data. For this purpose, several methods for discovering associative rules by continuous values are provided that these methods are called quantitative associative rules. Quantitative associative rules are displayed as follows:

$$
\left(\mathrm{L}_{1} \leq \mathrm{i}_{1} \leq \mathrm{U}_{1}\right) \wedge\left(\mathrm{L}_{2} \leq \mathrm{i}_{2} \leq \mathrm{U}_{2}\right) \rightarrow\left(\mathrm{L}_{3} \leq \mathrm{i}_{3} \leq \mathrm{U}_{3}\right)
$$

Where $\mathrm{i}_{\mathrm{j}}$ represents the $\mathrm{j}$ th $\mathrm{item}, \mathrm{L}_{\mathrm{j}}$ and $\mathrm{U}_{\mathrm{j}}$ represent the lower band and upper band respectively.

Therefore the $\mathrm{j}$ th item can adopt the values in the range of $\left[\mathrm{L}_{j}, \mathrm{~L}_{j}\right]$.

\section{1-3-3-3 Discovering fuzzy association rules}

One of the main problems related to presented methods for discovering quantitative associative rules is sharp boundaries between successive intervals. For example consider traits such as age assuming values $(0,35)$ at young ages, $(35,65)$ in the middle ages and $(65,100)$ in the older classes of age. In this case, if a person who is in the 35th year of his birth will go to the young category but a day later, he will be considered is middle aged class. In fact, transition between being young or not is not sudden but gradual. So to model these concepts fuzzy sets are used instead of certain collections. According to fuzzy concepts that have already been proposed, for example, for the characteristic of age, a fuzzy set with elements of young, middle-aged and older is considered that each numeric value to age belongs to a degree of membership in each of above mentioned elements. In discovering fuzzy association rules, each item is considered as a linguistic variable. 
The linguistic variables are variable with the values of vocabulary or sentences in a natural language. An item with little value can be divided to $\mathrm{k}$ different language values that $\mathrm{k}>1$. Such as age characteristic that three values of young, middle aged and old was defined for it. In the fuzzy associative rules discovery process, first a small amount each of the items is needed in each of the transactions to be converted to a fuzzy set. This is done by means of membership functions that can be given as an input to the user or the clustering methods for automatic conversion of transaction data sets to the corresponding fuzzy membership functions can be used.

The overall form of fuzzy association rules is as follows:

$$
\begin{aligned}
\text { If } \mathrm{X} & =\left\{\mathrm{x}_{1}, \mathrm{x}_{2}, \ldots, \mathrm{x}_{\mathrm{p}}\right\} \text { is } A=\left\{f_{1}, \mathrm{f}_{2}, \ldots, \mathrm{f}_{\mathrm{p}}\right\} \\
\text { Then } \mathrm{Y} & =\left\{\mathrm{y}_{1}, \mathrm{y}_{2}, \ldots, \mathrm{y}_{\mathrm{q}}\right\} \text { is } \mathrm{B}=\left\{g_{1}, \mathrm{~g}_{2}, \ldots, \mathrm{g}_{\mathrm{q}}\right\}
\end{aligned}
$$

where $\mathrm{X}$ and $\mathrm{Y}$ show two sets of items that $\mathrm{X} \cap \mathrm{Y}=\varnothing . \mathrm{A}$ and $\mathrm{B}$, respectively, indicate the fuzzy sets corresponding to $\mathrm{X}$ and $\mathrm{Y}$ items. For example $f_{l}$ is the fuzzy set related to $x_{l}$ item.

\section{1-3-4-Finding sequential patterns}

For modeling and predicting the behavior of the user we need to make models for his behavior and activities that are done sequentially. What we want to do is what occurs in the future.

\section{1-3-4-1- The N-gram model}

Here an N-gram based model is provided that is used to predict the next question of the user. $\mathrm{N}$-gram model is suitable to model and predict the behavior of a person based on his past performances. This method is applicable in a wide range of programs on the web, including sending the required information by the user and extracting information or helping the users.

\section{1-3-4-2- The Markov model}

Markov model is a model suitable for modeling and forecasting the behavior of the user while surfing the web. In general, the input is a sequence of web pages that have been viewed by the user and the goal is to build a Markov model that can be used to predict future visits to pages.

\section{1-3-4-3 predicting using the detailed adjustment}


A good way to describe this model is using the tree. Each node of the tree shows a sequence of actions. These actions will be determined by scrolling the tree from the root to that node. For each level of the tree a basic node is considered. For example, if we are on the basic level, basic node at this level represents the action that occurred last in sequence of actions. A tree with depth (the depth of the node at root is considered zero) in fact shows a Markov n-1order model.

\section{1-3-4-4 Predictions based on the suffix tree}

Suppose that the $\mathrm{s}^{\mathrm{n}}{ }_{1}=\mathrm{s} 1 ; \mathrm{s} 2, \ldots . \mathrm{sn}$ sequence is a set of events. Each symbol of $\mathrm{s}^{\mathrm{n}}{ }_{1}$ belongs to a limited set of alphabet letters. The goal is to predict $S_{n+1}$. The symbol that is presented as anticipate is one that has appeared more than others in points that are stored as indices.

\section{1-3-4-5 Predicting based on a probable postfix tree}

In tree-based predicting algorithm based on a probable postfix tree, as well as the ppm method a maximum length is considered for the sequences history. The algorithm tries to provide the best prediction model according to the limitations. The value of this limitation is considered as D. Each edge has a specific symbol of $\Sigma$. Suppose $s^{n}{ }_{1}$ is a sequence. The algorithm sets up a set of prefixes first, in a way that each sub sequence of $S$ is shorter than $\mathrm{D}$ and the number of iterations is less than a threshold value. In the second phase, 2-requisite must be checked for any sequences in the set of $\mathrm{S}$. If these sequences have satisfied the requisite, all the prefixes are located in pst. These two requisite include:

The sequence of $S$ must be meaningful for a symbol such as $\alpha$. It means that $\mathrm{p}(\alpha 1 \mathrm{~s})$ is greater than a threshold value of $\mathrm{P}_{\min }$. Sequence of $\mathrm{S}$ has more of his father's information to predict the symbol $\alpha$ after the s.

\section{CONCLUSION}

Today's search engines offer search results regardless of the user's interests or career fields. Accordingly, when users use search engines often encountered with results that may be not be of interest to them. What is presented in this paper is an idea that by recognizing the user as well as his historical behavior actions can be taken to improve the ranking (Ranking) of search results in a way that what is more important for the user and more in tune with his interests are placed at a 
higher rank. In this article, we examined several approaches to personalize web pages that what is new in this paper is an idea of how to identify patterns of user behavior and the basis is using data mining techniques in order to interpret the meaning of the pages of the user visits. Search engines rank and recover web pages based on their similarity to the users' questions.

\section{REFERENCES}

[1] Achananuparp P., Han H., Nasraoui O.and Johnson R., "Semantically Enhanced User Modeling”, Proceedings of the 2007 ACM Symposium on Applied Computing (Seoul, Korea, March 11 - 15, 2007). SAC '07. ACM, New York, NY, 1335-1339.

[2] Nasraoui O., Soliman M., Saka E., Badia A.and Germain R., "A Web Usage Mining Framework for Mining Evolving User Profiles in Dynamic Web Sites", IEEE TRANSACTIONS ON KNOWLEDGE AND DATA ENGINEERING, 2008, vol. 20, $1041-4347$.

[3] Eirinaki M.and Vazirgiannis M., "Web Mining for Web Personalization", ACM Transactions on Internet Technology, 2003, 3, 1-27.

[4] Pierrakos D., Paliouras G., Papatheodorou C.and Spyropoulos C. D., "Web Usage Mining as a Tool for Personalization: A Survey”, User Modeling and User-Adapted Interaction, $2003,13,311-372$.

[5] Dai H. and Mobasher B., "Integrating Semantic Knowledge with Web Usage Mining for Web Personalization", Web Mining: Applications and Techniques, Idea Group Publishing, 2005, 276-306.

[6] Agrawal R., Imielinski T., and Srikant R., "Mining Association Rules Between Sets of Items in Large Databases ," in proceedings of the ACM SIGMOD International Conference on Conference on Management of Data, pp. 207-216, 1993.

[7] techniques, Volume 18 Issue 3, July, 2004.

[8] ZANGIAN M., HASHEMI SMR., YAGHMAEE F., and MOSHTAGH E. "COMPARATIVE EVALUATION OF FACE RECOGNITION ALGORITHMS USING 
AND NONINDIVIDUAL ALGORITHMS, Vol 2, Issue1 2014 , pp 16-19.( Special Online Issue-February 2014)

[9] Mohammadpour M., Hashemi SMR.and Broumandnia A., "Improve Image Re-targeting Algorithm Using Markov Random Field", The second international conference on Pattern Recognition and Image Analysis, 2015.

[10] Hashemi SMR., Kalantari M., and Zangian M., "Giving a New Method for Face Recognition Using Neural Networks.", International Journal of Mechatronics, Electrical and Computer Technology, 2014, 4(11), 744-761.

[11] Hashemi SMR., Zangian M., Shakeri M., and Faridpoor M., "Survey Article about Image Fuzzy Processing Algorithms."The Journal of Mathematics and Computer Science, 2014, 13(1), 26-40.

[12] Hashemi SMR., Broumandnia A., Kashian M.and Moshtagh E., "A Survey on the Methods of Face Detection." INTERNATIONAL JOURNAL OF CURRENT LIFE SCIENCES, 2014, 4(12), 13367-13370.

[13] Hashemi SMR., Zangian M., Shakeri M., and Pak Aghideh A. "Evaluating and classification of face detector algorithms, Applied Mathematics in Engineering, Management and Technology 2 (2), 385-388.

[14] Hashemi SMR., "Review of algorithms changing image size " Cumhuriyet Science Journal, 2015, 36(3), Special Issue 2015.

[15] Hashemi SMR., Broumandnia A. "A Review of Attention Models in Image Protrusion and Object Detection."The Journal of Mathematics and Computer Science ,2015, 15(4), 273-283.

[16] Hashemi SMR., Broumandnia A., "A New Method for Image Resizing Algorithm via Object Detection ."International Journal of Mechatronics, Electrical and Computer Technology, 2015, 5(16). 213-234.

[17] Hashemi SMR., Mohammadalipour S.and Broumandnia A., "Evaluation and classification new algorithms in Image Resizing.", International Journal of Mechatronics, 
Electrical and Computer Technology, 2015, 5(18) Special Issue, Dec. 2015, PP. 2649-2654, ISSN: 2305-0543

[18] Hashemi SMR., Faridpoor M. "Evaluation of the Algorithms of Face Identification" IEEE-2015, 2nd International Conference on Knowledge-Based Engineering and Innovation (KBEI).

[19] Hashemi SMR. "A Survey of Visual Attention Models" Ciência e Natura, v. 37 Part 2 2015, p. 297-306 ISSN impressa: 0100-8307 ISSN on-line: 2179-460X.

[20] Deramgozin M. M., Hashemi S., Fard A. B. "Face Recognition Improvement in Angled Status Using Invasive Weed Optimization Algorithm And fuzzy System" 2016 1st International Conference on New Research Achievements in Electrical and Computer Engineering (2016).

[21] Hashemi SMR., A. Broumandnia, Z. Zangian And E. Moshtagh "ANNOTATING THE IMAGES USING BACKGROUND"Indian Journal of Scientific Research, Special Online Issue-April 2014.

[22] Hashemi SMR, Zangian M, Moshtagh E, Zangian Z. "Forecasting daily electrical energy delivered to the electric power distribution company using intelligent methods" Applied Mathematics in Engineering, Management and Technology, 2014, 2(2), 409-414.

[23] Nouhi A., Hashemi SMR, Yaghmaee F, Zangian M. "Indexing for PERSIAN Textual Images " Applied Mathematics in Engineering, Management and Technology 2 (1) (Jan 2014)

[24] Hashemi SMR., Hajighorbani M., Deramgozin M. M. and Minaei-Bidgoli B., "Evaluation of the Algorithms of Face Identification ", International Journal of Mechatronics, Electrical and Computer Technology, 2016, 6(19), 2016

[25] Hashemi SMR., Deramgozin M. M., Hajighorbani M.and Broumandnia A. "Methods of Image Re-targeting Algorithm Using Markov Random Field ", International Journal of Mechatronics, Electrical and Computer Technology, 2016, 6(20), 2016 
[26] HajiGhorbani M., Hashemi SMR., Minaei-Bidgoli B., Safari S. "A Review of Some Semi-Supervised Learning Methods "IEEE-2016, First International Conference on New Research Achievements in Electrical and Computer Engineering 2016.

[27] Deramgozin MM., Hashemi SMR., BastanFard A., HajiGhorbani M."Face Recognition Improvement in Angled Status Using Invasive Weed Optimization Algorithm And fuzzy System" IEEE-2016, First International Conference on New Research Achievements in Electrical and Computer Engineering 2016.

[28] Hashemi SMR., Hajighorbani M., Deramgozin MM., Minaei-Bidgoli B., "The Comparison of Face Detection Methods in Angled Mod" IEEE-2016, First International Conference on New Research Achievements in Electrical and Computer Engineering 2016.

[29] Hashemi SMR., Deramgozin MM., Hajighorbani M., Minaei-Bidgoli B."A review of the methods of watermarking in digital texts" IEEE-2016, First International Conference on New Research Achievements in Electrical and Computer Engineering 2016.

\section{How to cite this article:}

Hajighorbani M, Reza Hashemi SM, Deramgozin MM, Faridpour M. Mehdi Khoshsima Shahraki E, Esmaeil Azimi Khatibani F and Jafar Shahraki G. A study on the personalization methods of the web. J. Fundam. Appl. Sci., 2016, 8(2S), 1026-1045. 